\title{
BRECHA DE IMPLEMENTACIÓN DEL E-GOBIERNO EN EL HOSPITAL REGIONAL DE MOQUEGUA
}

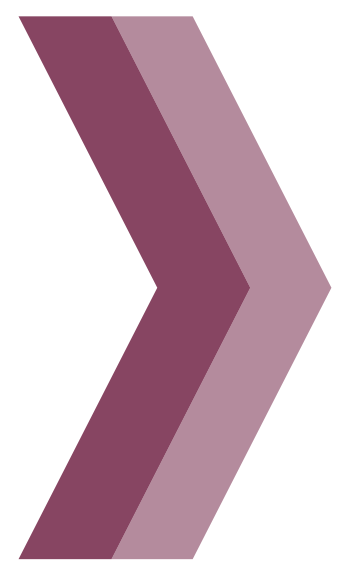

\section{GAP OF IMPLEMENTATION OF THE E-GOVERNMENT IN THEREGIONAL HOSPITAL OF MOQUEGUA}

Irwing Aspajo Grandez ${ }^{1}$ Anthony Dickson Villegas Flores ${ }^{2}$

1. Doctor en Administración por la Universidad Nacional de San Agustín, Jefe de la Oficina de Planeamiento Estratégico del Hospital Regional de Moquegua.

2. Estudiante del X Ciclo de la Escuela Profesional de Gestión Pública y Desarrollo Social. Universidad Nacional de Moquegua.

\section{RESUMEN}

INTRODUCCIÓN: El gobierno electrónico es una herramienta de enorme potencial en términos de la Administración Pública. El objetivo fue identificar la brecha de la implementación de e-gobierno en el Hospital Regional de Moquegua. MATERIAL Y MÉTODOS: Investigación de tipo descriptivo, cualitativa, se realizó entre los meses de junio a octubre del año 2017, en la provincia de Mariscal Nieto, región Moquegua, Perú. Se utilizó información secundaria cuantitativa y cualitativa oficial. Las variables de estudio consideradas para alcanzar el nivel de implementación de e-gobierno, fueron: mecanismos de comunicación interna, eservicios y gestión de información. RESULTADOS: Resulta necesario la digitalización de la gestión documentaria, que conlleve al mejoramiento de los procedimientos de carácter interno que beneficiarán transversalmente en el servicio. Por su parte, existen algunos servicios que presta el nosocomio que podrían brindarse parcialmente utilizando las tecnologías de la información y comunicación; como, por ejemplo, Consulta Externa, la cual podría ser diligenciada en parte presencial y virtual, dada la naturaleza presencial de los servicios de salud hospitalario. Se requiere una gestión dedicada que genere cambios en el manejo de la información, ya que de ello depende la toma de decisiones gerencial, teniendo en cuenta que esto conlleva la implementación de una cultura de trabajo eficiente con procesos estandarizados, acción que depende del sinceramiento de la información. CONCLUSIÓN: No se utiliza las bondades de la implementación del e-gobierno; por tal motivo, no se optimizan los recursos: tiempo, materiales y humanos, que generen reditúo social, que sea diferenciado y sostenible con el objetivo de proporcionar el valor público que la ciudadanía espera.

PALABRAS CLAVES: Hospital, E-gobierno, Valor Público

\section{ABSTRACT}

INTRODUCTION: The objective is to identify the gap in the implementation of e-government in the Regional Hospital of Moquegua. Descriptive, qualitative research was conducted between the months of June to October of the year 2017, in the province of Mariscal Nieto, Moquegua region, Perú. MATERIAL AND METHODS: Qualitative quantitative and qualitative secondary information was used. The study variables considered to reach the level of e-government implementation were: internal communication mechanisms, e-services and information management. RESULTS: From there view, it is identified that the digitization of the documentary managemen tis necessary, which leads to the improvement of internal procedures that will benefit transversally in the service. On the other hand, there are some services provided by the hospital that could be partially provided using information and communication technologies; as, for example, External Consultation, which could be completed in person and virtual part; given the face-to-face nature of the hospital health services. And, finally, a dedicated management is required that generate schanges in the handling of the information, since; it depend son managerial decision making; taking into account that this entails the implementation of an efficient work culture with standardized processes, action that dependson the disclosure of information. CONCLUSIONS: The benefits of the implementation of e-government are not used, for this reason, resources are not optimized: time, material and human, that generate social return, that is differentiated and sustainable, in order to provide value public that the public expects.

KEYWORDS: Hospital, E-government, Value Public 


\section{INTRODUCCIÓN}

El gobierno electrónico es una herramienta de enorme potencial en términos de la Administración Pública. Específicamente, porque permite incrementar la calidad de los servicios públicos, mejorar el proceso de toma de decisiones y promover una mayor participación ciudadana durante el ciclo de gobierno. Marca un paso fundamental en la transición hacia la Sociedad de la Información en tanto actúa como agente promotor de la alfabetización digital y la universalización del acceso a las nuevas Tecnologías de la Información y Comunicación (TIC).Por lo que se ha procedido a dividir en tres componentes de desarrollo de tecnologías de la información y comunicación: mecanismos de comunicación interna, e-servicios y gestión de información, para poder medir el nivel de implementación de E-gobierno en el Hospital Regional Moquegua.

\section{MATERIAL Y METODOS}

El estudio es de tipo descriptivo, cuyo método es de investigación cualitativa; dado que se usa un marco referencial teórico de los componentes que debe contar un sistema E-gobierno en el Hospital Regional de Moquegua, que para efectos del estudio nos referiremos como Hospital. El material utilizado es información secundaria cuantitativa de carácter oficial.

En preciso indicar que los niveles de comunicación interna se definen como los que se desarrollan como unidad ejecutora conformante del pliego Gobierno Regional de Moquegua; por lo que las relaciones que se mantienen con otras instancias como la Gerencia Regional de Salud, Sede Central Gobierno Regional Moquegua, Ministerio de Salud, Ministerio de Economía y Finanzas, Seguro Integral de Salud, entre otras entidades de los tres niveles de gobierno en el estado peruano, serán consideradas de carácter externo. El mismo criterio se aplicará para analizar el componente de e-servicios y gestión de información hospitalaria.

Comunicación Interna: entendida como las actividades que se realizan dentro de la entidad para mantener relaciones laborales entre los miembros de la empresa por medio de la circulación de mensajes que se originan a través de los diversos medios de comunicación empleados por ella, con el objetivo de proveer comunicación. Ahora bien, la organización analizada, representa una entidad del Estado que basa su gestión en la administración burocrática; modelo de organización que obedece a las conductas racionales para alcanzar objetivos enfatizando el carácter legal de las normas y reglamentos que brinda el carácter formal de las comunicaciones; donde las reglas, decisiones y acciones administrativas se formulan y registran por escrito, buscando que los procedimientos se estandaricen y generando el cumplimiento del principio Tayloriano de jerarquización de la autoridad. Ejerciendo, de esta forma, el control en todo el proceso administrativo.Ello, en el Hospital, se viene desarrollando de manera escrita utilizando documentos formales como lo son: Informes, Memorándums, Cartas, Resoluciones Administrativas, Resoluciones Ejecutivas Directorales. No obstante, se utilizan correos electrónicos personales para comunicar, que incluye envío de información, aspectos laborales y de utilidad para la generación de los documentos impresos. Así tenemos: Informe, que comunica acciones realizadas, solicita aprobación de propuestas, la autorización para determinada acción, transmite opiniones técnicas, comunica actos y/o acciones de prevención, se remite al superior jerárquico; centraliza la toma de decisiones en el titular de la entidad, (por efectos de miedo al control posterior, no existe formato que estandarice su formulación). Memorando, dispone orden a subordinado, convoca a reunión, formaliza llamada de atención, establece coordinaciones entre órganos estructurados del mismo nivel jerárquico, se utiliza como medio formal para ejercer autoridad, (no existe formato que estandarice su formulación), Resoluciones Administrativas, formaliza actos de administración en las cuatro unidades orgánicas a cargo de la Oficina de Administración, (no existe formato que estandarice su formulación), no obedece a opiniones o informes técnicos que motiven su elaboración, se basa en aspectos normados por el Gobierno Nacional, no se adecúa la norma a la realidad hospitalaria. Resoluciones Ejecutivas Directorales, formaliza actos de administración y asistenciales de alcance a todos los órganos estructurados del hospital, (no existe formato que estandarice su formulación), no obedece a opiniones o informes técnicos que motiven su elaboración, se basa en aspectos normados por el Gobierno Nacional. Cartas y/o Solicitudes, formaliza requerimientos de los trabajadores que devienen de obligaciones del empleador o exigencias de derechos laborales, (no existe formato que estandarice su formulación). Formatos Administrativos, solicitudes de afectación de caja chica, viáticos, vacaciones, papeletas de salida y otros, son generados por la administración para evidenciar actos regulares de la gestión. Proveídos, escritos que se realizan a mano, a 
fin de solicitar, autorizar y disponer acciones a otros órganos estructurados, se evidencia abuso en su utilización, se formulan con la finalidad de eludir responsabilidades y la no generación de la formalización de actos a nivel de subordinación. De lo analizado, podemos determinar que si bien, la administración burocrática establece un modelo que sostiene la formalización de la comunicación interna a través de normas y reglas que debieran ser de carácter escrito; teniendo en cuenta que esta escuela data de los años 40, es decir cuenta con 67 años de vigencia; pero que ella puede ser complementada con diferentes mecanismos que utilicen las tecnologías de la información y comunicación que conlleven necesariamente a establecer la gestión por procesos; los cual implicaría una serie de cambios al eliminar y/o suprimir procedimientos que generen valor en la comunicación interna. Todo ello con el objetivo de implementar estándares de eficiencia, entendida como la optimización de recursos: Económicos, Materiales y de Tiempo para que estos aspectos administrativos puedan ser eficaces en la prestación del servicio de salud.

E-servicios: servicio, considerada como cualquier actividad o beneficio que una parte puede ofrecer a otra,básicamente es intangible y no tiene como resultado la propiedad de algo. Cuenta además con las siguientes características: intangibilidad, ya que estos no se pueden ver, degustar, tocar, oír ni oler antes de la adquisición; variabilidad, debido a que su calidad depende de quién los presta, cuándo, dónde y cómo se prestan; inseparabilidad, porque no pueden separarse de sus proveedores; y por último, caducidad, puesto que no pueden almacenarse para su venta o uso posterior. Partiendo de ello, podemos conceptualizar el E-servicios como la forma en que las tecnologías de la información y comunicación optimizan la prestación de un servicio o forman parte del mismo para la entrega del servicio en las organizaciones, específicamente utilizando el internet como base para operativizar y concretar el mismo. El desarrollo del E-servicios en la administración pública en el Perú ha venido mejorando a razón del comportamiento y cambio del mismo en los ciudadanos para con la respuesta positiva en el uso de las tecnologías de la información y comunicaciones para obtener un servicio de las entidades públicas; los cuales contribuyen de manera eficiente en la generación de confianza entre la ciudadanía y el Estado. Así tenemos, por ejemplo: Operaciones en Línea de la Superintendencia Nacional de Administración Tributaria SUNAT; el Sistema Electrónico de Contrataciones del Estado SE@CE del
Organismo Supervisor de Contrataciones del Estado OSCE; el Voto Electrónico, Búsqueda de lugares de Sufragio de la Oficina Nacional de Procesos Electorales ONPE; Seguimiento de Trámite Documentario STD Ministerio de Economía y Finanzas; Tributación en Línea, de la Municipalidad de Miraflores; Bolsa de trabajo en línea. Empleos Perú, del Ministerio de Trabajo y Promoción del Empleo; Alerta Registral Superintendencia Nacional de Registros Públicos SUNARP; Infobras del Ministerio de Economía y Finanzas MEF, el Sistema Electrónico de Notificaciones Judiciales del Poder Judicial; Multired Virtual, del Banco de la Nación; entre otras iniciativas. Como podemos apreciar, existen varias acciones que se han concretado por diferentes entidades públicas y que han utilizado las tecnologías de la información y comunicación, vinculadas al uso del internet, las cuales generan interacción con la ciudadanía que obtiene beneficios directos. Ello representa el constructo que se define en el presente estudio, en lo concerniente al E-servicio en las instituciones públicas; puesto que en futuras investigaciones podríamos plantear o adaptar un modelo de medición a razón de la interacción entre el servicio del Estado y la ciudadanía bajo la conceptualización de la co-creación, entendida como la participación del ciudadano en la generación del servicio utilizando la big data que genera el Estado. Todo ello considerando que los ejemplos detallados solo representan una parte de todas las implementaciones realizadas sobre el particular a la fecha en el Estado peruano. Es preciso indicar que la medición de la utilización de las tecnologías de la información y comunicaciones en la generación de eservicio en el estado se realiza en dimensiones como: democracia, transacción, interacción y acceso a la información; relacionado a las bondades que se operan a través de los portales web institucionales. Ahora bien, en el Hospital, no se cuenta a la fecha con ninguna iniciativa de vincular los servicios que presta a la ciudadanía directa e indirectamente con el uso de tecnologías de la información y comunicación, en participación con la ciudadanía; salvo el Portal de Transparencia, que obedece al cumplimiento de normas establecidas por el Gobierno Nacional. Para entender mejor lo señalado, definiremos los servicios que brinda el Hospital a la ciudadanía, en base a la normatividad vigente. Servicios de Atención Directa al Ciudadano. Consulta Externa, organizada para la atención integral, de acuerdo a la cantidad de profesionales de la salud especializados con que se cuenta, a usuarios que no estén en condición de urgencia y/o emergencia. Centro Quirúrgico, organizada para realizar procedimientos 
anestesiológicos e intervenciones quirúrgicas, así como para la atención de la fase de recuperación post anestésica. Emergencias, organizada para la atención inmediata y permanente a usuarios que estén en condición de urgencia y/o emergencia. La atención debe estar organizada por prioridades de acuerdo a lo establecido en la norma técnica de salud (I, II y III), se debe garantizar la disponibilidad de los servicios de apoyo al diagnóstico y tratamiento necesarios. Hospitalización, se organiza para brindar los cuidados necesarios a los usuarios que requieran permanencia y necesidad de soporte asistencia por doce (12) horas, por su grado de dependencia o riesgo. Los cuales están referidos a las atenciones y procedimientos médicoquirúrgicos, con fines diagnósticos, terapéuticos o de rehabilitación. Servicios de Apoyo al tratamiento, servicio de procesamiento e interpretación de los estudios por radiaciones ionizantes y no ionizantes. Servicio de toma, recepción, procesamiento de las muestras biológicas y emisión de resultados de los procedimientos de Patología Clínica. Servicio de realización de exámenes anatomo patológicos para confirmar, esclarecer o definir diagnósticos, garantizando la calidad y oportunidad de los informes. También incluyen los servicios que se realizan en los órganos estructurados de Farmacia, Nutrición, Psicología, Servicio Social, Banco de Sangre y Central de Esterilización. Servicios de Unidad de Cuidados Intensivos - UCl, organizada para la atención de salud especializada en medicina intensiva a usuarios críticos en condición de inestabilidad y gravedad persistente. Esta atención debe garantizarse las 24 horas del día, se divide para la atención en servicios de cuidados intensivos generales, neonatales y pediátricos. Servicios de Atención Indirecta al Ciudadano. Consulta de Tarifas Hospitalarias, información sobre las tarifas que cobra el Hospital a pacientes no subsidiados por el Estado: SOAT, convenios con seguros particulares, convenios con empresas privadas, SALUPOL y los pacientes que acuden al nosocomio y no cuentan con ningún tipo de seguro o manifiestan su deseo de ser atendidos particularmente. Libro de Reclamos y Quejas, en el cual el ciudadano manifiesta su reclamo (al servicio brindado) o queja (dirigida al mal trato de un trabajador de la entidad de manera personalizada). Selección de Personal, Proceso administrativo de carácter público para incorporar a personal para que labore en el Hospital. Generación de Información, Generación de información útil para el ciudadano referida a las diferentes enfermedades que adolece la población que acude al hospital, así como el acceso a información de índole epidemiológica. Portal de Transparencia, Acceso a información de carácter interna administrativa del Hospital, sobre la gestión de los recursos asignados para su gestión y que representa de interés público. Por consiguiente, y debido a la naturaleza de los servicios que brinda el Hospital, es que no todos pueden ser vinculados como componente principal al E-servicios: entendido como la construcción del mismo con participación del ciudadano. No obstante, se podría implementar en dos (02) servicios de atención directa: Consulta Externa y Servicios de Apoyo al Tratamiento y Diagnóstico; por su parte en los servicios de atención indirecta podrían ser incorporados los siguientes servicios: Consulta de Tarifas Hospitalarias, Libro de Reclamos y Quejas, Selección de Personal y Acceso a Información Hospitalaria; debido a que el Portal de Transparencia sí se encuentra desarrollado, a razón de exigencias de entes superiores. Se debe precisar que ello no impide que se utilicen tecnologías de la información y comunicación en el desarrollo de los servicios hospitalarios, pero que estos no se realizan con participación del ciudadano, sino funcionan como parte del servicio diligenciado por el personal del Hospital; así tenemos: Realización de procesos de adquisiciones y contrataciones utilizando la plataforma web SE@CE, con vinculación entre la entidad y los proveedores. Tecnologías de procesamiento de información a partir del procesamiento de muestras de sangre, orina y otros fluidos a través del equipo automatizado de Laboratorio. Intervenciones quirúrgicas utilizando equipos biomédicos digitalizados con tecnologías de ópticas de alta definición, como las bondades que ofrece la Torre Laparoscópica o el Electro bisturí y el Arco en C. Tecnologías de procesamiento de información a partir de la toma de placas radiográficas por intermedio del equipo de Rayos X. Utilización de tecnologías PLC, Controladores Lógicos Programables que emiten datos para la toma de decisiones del personal de salud, en equipos como el esterilizador y las autoclaves; entre otras tecnologías de la información y comunicaciones que se desarrollan de manera interna en la prestación del servicio de salud.

Gestión de Información Hospitalaria. La información hospitalaria es la que generan las diversas Unidades Productoras de Servicios de Salud UPSS; las cuales se registran en los aplicativos informáticos que cuentan con un enfoque de control concurrente y posterior; no obstante, los mismos usuarios de dichos aplicativos desconocen las bondades que la data puede brindar a nivel de información para la toma de decisiones, ya que esta se registra a nivel de cumplimiento y luego no se procesa, analiza ni sistematiza su uso. Ahora bien, la 
información hospitalaria debe ser utilizada por el equipo directivo del nosocomio para la toma de decisiones a nivel de priorización de los esfuerzos hacia determinada tendencia, es decir, determinar recursos (Humanos, Materiales y de Tiempo) a fin de satisfacer las necesidades de los usuarios del servicio de salud. No obstante, esto no sucede exactamente; ya que la toma de decisiones de gestión se encuentra centralizada por el Gobierno Nacional (Ministerio de Salud, Ministerio de Economía y Finanzas, Seguro Integral de Salud y Contraloría General de la República): Esto se debe a que los aplicativos informáticos cuentan con sesgo de control y las normas que se han venido emitiendo son restrictivas, tanto de gasto como de actuación para el equipo directivo. Por lo que, pasaremos a ilustrar lo antes señalado con dos ejemplos: Uno referido al actuar administrativo y el segundo a la parte asistencial, ejemplo de índole administrativo. El Sistema Integrado de Administración Financiera SIAF-SP cuenta con tres módulos: Procesos Presupuestarios, Administrativo y Contable. Uno de los productos de vital importancia es el Estado de Resultados; los cuales reflejan un simple registro de información de ingresos y egresos a nivel presupuestario y financiero, es decir, no se utiliza para reflejar el nivel de cumplimiento de las metas institucionales con el presupuesto asignado; de reflejar esto, en algún estado financiero, sería sinónimo de mala gestión; ya que lo habitual es cumplir con los objetivos de la asignación presupuestal histórica ante la mirada fiscalizadora y sancionadora del Gobierno Nacional. En síntesis, la información que se registra en el SIAF-SP sirve para la toma de decisiones efectiva del Gobierno Nacional (Ministerio de Salud, Ministerio de Economía y Finanzas, Seguro Integral de Salud y la Contraloría General de la República) con ánimos de supervisión y fiscalización; pero no de mejora continua de los servicios. Y el nivel de actuación del equipo de gestión hospitalaria es de alcance regional, donde se pierde el interés por la problemática presupuestal en la prestación de servicios asistenciales en Hospitales. Ejemplo de índole asistencial. El Aplicativo Informático de Funciones Obstétricas y Neonatales FON fue creado por el Ministerio de Salud a fin de registrar el estado de infraestructura, equipamiento, personal y de prestación de servicios en la Unidades Productoras de Servicios de Salud UPSS; que brindan y desarrollan funciones obstétricas y neonatales en los establecimientos de salud. No obstante - a pesar de que cada periodo solicitado por el Gobierno Nacional, se registra la información en cada módulo del aplicativo y se reflejan falencias en los componentes que devienen en asignación de recursos y optimización de los mismos - esta data no es procesada a nivel de información por los entes decisorios financieros en el nosocomio, Gobierno Regional y Gobierno Nacional; porque las reglas fiscales no permiten demandas adicionales de presupuesto. En síntesis, el registro de información en este aplicativo no sirve para la mejora continua de los servicios prestados, puesto que el nivel de priorización de los recursos no es de dominio del equipo de gestión hospitalaria; por lo que se encuentran limitados en una actuación que devengue en articulación de planeamiento y presupuesto. En ese sentido, podemos concluir que la generación de información hospitalaria es de utilidad para brindar el soporte a la toma de decisiones a nivel de Gobierno Nacional, bajo el enfoque de control y la concretización u operativización de las normas establecidas que generan centralización.

\section{RESULTADOS Y DISCUSIÓN}

Se detallan los resultados y acciones concretas que se debieran implementar en cada variable:

Comunicación interna. Las acciones que generarán valor en la emisión de los diferentes mecanismos de comunicación interna, utilizando tecnologías de la información y comunicación, se centran en el desarrollo de aplicación informática que estandarice la formulación de la documentación, digitalizando los documentos y generando el archivo digital del nosocomio. Ello debe estar acompañado del desarrollo de una Directiva que regule su utilización, con ello se ahorrará tiempo en la elaboración de documentación y mitigará el riesgo de pérdida de la información. A esto se suma el desarrollo de medidas de eco eficiencia y de una cultura de acceso a la información. En este espacio, es de suma importancia el uso y manejo del correo electrónico como herramienta oficial de comunicación sea esta horizontal y/o vertical dentro de la estructura organizacional, formalizando el principio administrativo de delegación de autoridad para determinar acciones a nivel de subordinación.

E-servicio. La implementación se debiera realizar con participación del ciudadano en algunos procesos que genera el nosocomio, catalogándolos como de intervención parcial en el desarrollo de la prestación asistencial: Consulta Externa. Solicitud de cita a través del portal institucional y/o aplicación para teléfonos inteligentes. Acceso on line a la programación de turnos de especialidades de los profesionales de la salud, detallando datos como: colegiatura, registro de especialidad y otros de relevancia para el ciudadano. 
Con ello el ciudadano podrá imprimir la cita o mostrarla en un teléfono inteligente para ser leído por un escáner y acceder a los siguientes procedimientos presenciales del servicio de consulta externa. Trámites para la emisión de Certificados Médicos, Informes Médicos, Certificados Psicológicos, Certificados de Discapacidad, entre otros. El ciudadano a través del Portal Institucional y/o Aplicación para móviles inteligentes podrá solicitar el inicio del trámite registrando los datos necesarios, donde se le generará un código de solicitud. Se acerca al Hospital, realiza los pagos según código reservado e inicia el trámite presencial. Cada emisión de Certificados deberá cumplir con los requisitos preestablecidos por el Ministerio de Salud, con ello se facilita el inicio del trámite utilizando la tecnología del Internet. Presentación de Reclamos y Quejas. Implementar el registro de reclamos y quejas vía acceso por el Portal Web Institucional. Generando un código para el seguimiento y notificación del trámite. Se facilita al ciudadano la celeridad de atención de sus requerimientos. Selección de Personal. Utilizando una plataforma virtual, implementar la estandarización de los procesos de selección de personal y llevarlos a cabo a través del portal institucional. Solo los ganadores del proceso presentarán los documentos que acreditan el manifiesto que tendrá valor de declaración jurada. Los procedimientos que estarían inmersos son: convocatoria, registro de postulantes, donde se establecen Declaraciones Juradas on line para garantizar la fidelidad de lo descrito como hoja de vida. Filtro de postulantes aptos, utilizando una aplicación que genere los resultados a razón de lo registrado por el postulante. Examen Psicológico generando una aplicación que en tiempo real emita el resultado del test. Examen de Conocimiento, con el manejo de una aplicación que en tiempo real emita el resultado del test. Entrevista Personal, con la utilización de Skype y/o WhatsApp; y por último el Resultado Final. El Hospital te informa. Utilización del Portal Web como medio informativo de la situación epidemiológica de las patologías que se atienden en el Hospital, generando espacios de acercamiento con la ciudadanía a través de la utilización de Facebook, Twitter o WhatsApp. Este tipo de espacios podrían ser el motor principal de la mejora de los servicios que presta el Hospital; ya que se apertura empatía con el ciudadano y co-creación en la prestación de salud. Se mantendría a la población informada sobre las principales dolencias que se intervienen en el Hospital y de las campañas médicas a realizarse. Como se logra apreciar, se pueden implementar el uso de tecnologías de la información por lo menos en cinco (05) servicios que brinda el
Hospital con participación del ciudadano a modo de interacción co-creativa, teniendo en consideración que esta sirve de complemento al servicio presencial de salud que se brinda; pues la presente busca identificar espacios donde el ciudadano colabora en la mejora de la prestación.

Gestión de la Información. La implementación de un aplicativo informático de generación de información hospitalaria que sea diseñado y/o adaptado con el propósito de apoyar en el correcto registro de información clínica - administrativa y la generación de información gerencial que permita una adecuada toma de decisiones, resulta de carácter neurálgico para todos los estamentos de la gestión hospitalaria. Haciendo más eficiente la gestión de los procesos operativos críticos del hospital - específicamente los de consulta externa, hospitalización, emergencia, archivo clínico y facturación-. Reduciendo los niveles de subfacturación y el tiempo de espera de los pacientes. Para lo cual es necesario liderar los siguientes cambios: Generar cultura de trabajo eficiente con procesos y modelos estandarizados, control económico y administrativo, automatización de procesos, mejora de tiempos de atención y sincerar información Hospitalaria. El Hospital, debido a la rustica gestión de la información que no es tomada en cuenta para la toma de decisiones, debe iniciar una integración de los aplicativos informáticos que generen procesamiento de datos hospitalarios; así tenemos por ejemplo los siguientes problemas identificados producto de una entrevista realizada a la Jefa de la Unidad de Gestión de la Calidad de dicho nosocomio .

De donde se concluye que, en la actualidad se cuenta con una base de datos de aproximadamente 80,000 pacientes; los cuales cuentan con Historia Clínica Física, sabiendo que existe un alto porcentaje de duplicidad en ellas, así como datos incompletos, donde tampoco se tiene identificado los tipos de Historias Clínicas como las judiciales, las activas, pasivas y las normales. Por lo que resulta necesario realizar una estrategia de depuración de Historias Clínicas física, dado los cambios de tecnología, y se tiene que evolucionar a una Histórica Clínica Electrónica con el número de DNI como único identificador. Se evidenció que, tanto en el servicio de emergencia como en consulta externa, hay tiempos muertos en los que no hay personal para realizar los procesos administrativos como dar citas, atención, cobro, dispensar medicamentos, en horas de almuerzo o cambios de turno. Por lo que resulta necesario la implementación de un sistema integrado hospitalario donde se registra en tiempo real, no 
debiendo regularizarse la atención por la validación existente y que otorgan una veracidad de la información que se registra. No se brindan citas a pacientes por estrategias ni programas (VIH, tuberculosis, salud mental, CRED ni de otros similares) todos los registros se realizan en libros o cuadernos, no figurando en ningún sistema; salvo los que se envía a GERESA Moquegua o Ministerio de Salud para efectos de control. Actualmente los problemas en archivo clínico afectan la creación de pacientes nuevos y por ello, la creación de la carpeta o folder de la Historia Clínica Física, no coincidiendo lo generado en sistema ni lo físico creado, por ser sistemas diferentes; adicionalmente no existen reportes para poder realizar seguimiento a ubicación de Historias Clínicas, volviendo ineficiente dicho proceso en los movimientos.

Se concluye que el Hospital no utiliza las bondades del uso del Internet y el Intranet en los mecanismos de comunicación interna; lo cual hace que no se optimicen los recursos: tiempo, materiales y humanos. La naturaleza de los servicios que brinda el Hospital no permite que estos se presten de manera directa utilizando en su totalidad una plataforma de eservicios. No obstante, se puede implementar bajo el enfoque de procesos, que parte de ellos pueda ser generado a través del uso del Internet e Intranet, generando espacios de interacción con la ciudadanía que podrían considerarse como de co-creación. No se cuenta con un sistema único y realizado a la medida de sus necesidades que brinde información clínica que se articule con la información administrativa. La gestión hospitalaria debe priorizar esfuerzos que coadyuven a que las instancias regionales tomen en consideración la brecha identificada para que su tratamiento se convierta en un objetivo regional que pueda ser sustentando ante las instancias nacionales para su respectivo financiamiento.

\section{REFERENCIAS BIBLIOGRÁFICAS}

1. Laudon J. Sistemas de Información Gerencial. Pearson Educación. 2012 abril; pp. 2-33.

2. Cockerman W. Ensayo sobre la Reforma del Estado y Modernización de la Administración Pública. "Gobierno Electronico" E-Government en el Sistema de Compras y Contrataciones. 2015 agosto; pp. 2, 10-13.

3. PCM-Perú. Política Nacional de Modernización de la Gestión Pública. Diario El Peruano. 2013 febrero: pp. 8.

4. Kotler P,AR. Marketin versión para Latinoamérica. In. México: Pearson Educación; 2007. pp. 261-265.

5. Alujas ÁV. Innovación en la Gestión Pública y Open Government (Gobierno abierto): Una vieja nueva idea.Buen Gobierno. In. Madrid, España; 2010. p. 126.
6. Aisherhri M,DS. E-Goverment Fundamentals. In. Australia: Griffith University Australia; 2012. pp. 36 y 37.

7. Gonzáles Zabala M,GLE. Identificación de Factores que afectan el desarrollo de la Inclusión Digital. In.: Revista Virtual Universidad Catolica del Norte; 2015. pp. 182-187.

8. Salud Md. Norma Técnica de Salud "Categorias de Establecimiento de Salud". Diario, El Peruano. 2011 julio;pp.4657.

9. Fernandez E. Evaluación de Macroprocesos de SUSALUD. Unidad de Gestión de la Calidad, Hospital Regional de Moquegua, Perú. 2017 octubre; pp.1-13.

\section{Correspondencia}

Irwing Aspajo Grandez

irwing78@gmail.com
Fecha de recepción: 10 de mayo de 2018

Fecha de aceptación: 12 de junio de 2018 